\title{
RANDOM WALK DELAYED ON PERCOLATION CLUSTERS
}

\author{
FRANCIS COMETS $* * *$ AND \\ FRANÇOIS SIMENHAUS, ${ }^{* * *}$ Université Paris Diderot - Paris 7
}

\begin{abstract}
We study a continuous-time random walk on the $d$-dimensional lattice, subject to a drift and an attraction to large clusters of a subcritical Bernoulli site percolation. We find two distinct regimes: a ballistic one, and a subballistic one taking place when the attraction is strong enough. We identify the speed in the former case, and the algebraic rate of escape in the latter case. Finally, we discuss the diffusive behavior in the case of zero drift and weak attraction.
\end{abstract}

Keywords: Random walk in a random environment; subcritical percolation; anomalous transport; anomalous diffusion; environment seen from the particle; coupling

2000 Mathematics Subject Classification: Primary 60K37

Secondary 60F15; 60K35; 82D30

\section{Model and results}

Consider the graph of nearest neighbors on $\mathbb{Z}^{d}, d \geq 1$, and write $\boldsymbol{x} \sim \boldsymbol{y}$ when $\|\boldsymbol{x}-\boldsymbol{y}\|_{1}=1$. Here, $\|\cdot\|_{1}$ is the $\ell_{1}$-norm, though $|\cdot|$ denotes the Euclidean norm.

An environment is an element $\omega$ of $\Omega=\{0,1\}^{\mathbb{Z}^{d}}$. Environments are used to construct the independent, identically distributed (i.i.d.) Bernoulli site percolation on the lattice. We consider the product $\sigma$-field on $\Omega$ and, for $p \in(0,1)$, the probability $\mathrm{P}=\mathscr{B}(p)^{\otimes \mathbb{Z}^{d}}$, where $\mathscr{B}(p)$ denotes the Bernoulli law with parameter $p$. A site $x$ in $\mathbb{Z}^{d}$ is said to be open if $\omega_{x}=1$ and closed otherwise. Consider the open connected components (the so-called clusters) in the percolation graph. The cluster of an open site $\boldsymbol{x} \in \mathbb{Z}^{d}$ is the union of $\{\boldsymbol{x}\}$ with the set of all $\boldsymbol{y} \in \mathbb{Z}^{d}$ which are connected to $\boldsymbol{x}$ by a path with all vertices open. The cluster of a closed site is empty. We denote by $C_{\boldsymbol{x}}$ the cardinality of the cluster of $\boldsymbol{x}$.

It is well known that there exists a critical $p_{\mathrm{c}}=p_{\mathrm{c}}(d)$ such that, for $p<p_{\mathrm{c}}$, P-almost surely (P-a.s.), all connected open components (clusters) of $\omega$ are finite, though, for $p>p_{\mathrm{c}}$, there a.s. exists an infinite cluster. Moreover, it follows from [1] and [11] that, in the first case, the clusters size has an exponential tail: for any $p<p_{\mathrm{c}}$, there exists $\xi=\xi(p)>0$ such that, for all $\boldsymbol{x}$,

$$
\lim _{n \rightarrow \infty} \frac{1}{n} \ln \mathrm{P}\left(C_{x} \geq n\right)=-\xi
$$

In this paper we fix $p<p_{\mathrm{c}}$. Let $\ell=\left(\ell_{k} ; 1 \leq k \leq d\right)$ be a unit vector, and let $\lambda$ and $\beta$ be two nonnegative numbers. For every environment $\omega$, let $\mathrm{P}_{\omega}$ be the law of the continuous-time

Received 16 October 2007; revision received 13 May 2008.

* Postal address: Université Paris Diderot - Paris 7, UFR de Mathématiques, Case 7012, 75205 Paris Cedex 13, France.

** Email address: comets@math.jussieu.fr

*** Email address: simenhaus@math.jussieu.fr 
Markov chain $\boldsymbol{Y}=\left(Y_{t}\right)_{t \geq 0}$ on $\mathbb{Z}^{d}$ starting at 0 with generator $L$ given for continuous, bounded functions $f$ by

$$
L f(\boldsymbol{x})=K \sum_{\boldsymbol{e} \sim \mathbf{0}} \exp \left(\lambda \boldsymbol{\ell} \cdot \boldsymbol{e}-\beta C_{\boldsymbol{x}}\right)(f(\boldsymbol{x}+\boldsymbol{e})-f(\boldsymbol{x})),
$$

where we chose the normalizing constant $K$ as $K=\left(\sum_{\boldsymbol{e} \sim \mathbf{0}} \mathrm{e}^{\lambda \ell \cdot e}\right)^{-1}$ for simplicity. Given $\omega$, define the measure $\boldsymbol{\mu}$ on $\mathbb{Z}^{d}$ by

$$
\boldsymbol{\mu}(\boldsymbol{x})=\exp \left(2 \lambda \ell \cdot \boldsymbol{x}+\beta C_{\boldsymbol{x}}\right) .
$$

The random measure $\boldsymbol{\mu}$ combines a shift in the direction $\boldsymbol{\ell}$ together with an attraction to large clusters. Observe that the process $\boldsymbol{Y}$ admits $\boldsymbol{\mu}$ as an invariant, reversible measure. Markov processes having $\boldsymbol{\mu}$ as an invariant measure are of natural interest in the context of random walks in random environments. They describe random walks which have a tendency to live on large clusters, the attraction becoming stronger as $\beta$ is increased. The isotropic case, $\lambda=0$, has been considered in [14] with a different, discrete-time dynamic. There, the authors proved that the walk was diffusive for small $\beta$ and subdiffusive for large $\beta$. The investigation of slowdowns in the anisotropic case is then natural. In [16] a random resistor network was considered with an invariant, reversible measure of the form $C(\boldsymbol{x}, \omega) \mathrm{e}^{2 \lambda \boldsymbol{\ell} \cdot \boldsymbol{x}}$, where the random field $\left(C(\boldsymbol{x}, \omega) ; \boldsymbol{x} \in \mathbb{Z}^{d}\right)$ is stationary ergodic and bounded away from 0 and $+\infty$ : in this case the random walks in random environments are ballistic for all positive $\lambda$.

The study of a general dynamic in the presence of a drift contains many difficult questions, and the advantage of the particular process $\boldsymbol{Y}$ considered here is that we can push the analysis further. We could also handle the discrete-time analog of $\boldsymbol{Y}$, i.e. the random walks in random environments with geometric holding times instead of exponential holding times, which falls in the class of marginally nestling walks in the standard classification (see, e.g. [20]). The Markov process $\boldsymbol{Y}$ can also be described by its skeleton and jump rates. The skeleton $\boldsymbol{X}=\left(X_{n}\right)_{n \in \mathbb{N}}$ is defined as the sequence of distinct consecutive locations visited by $\boldsymbol{Y}$. Then, $\boldsymbol{X}$ is a discrete-time Markov chain with transition probabilities $\tilde{\mathrm{P}}$, given for $\boldsymbol{x} \in \mathbb{Z}^{d}$ and $\boldsymbol{e} \sim \mathbf{0}$ by

$$
\tilde{\mathrm{P}}\left(X_{n+1}=\boldsymbol{x}+\boldsymbol{e} \mid X_{n}=\boldsymbol{x}\right)=\frac{\mathrm{e}^{\lambda \boldsymbol{\ell} \cdot \boldsymbol{e}}}{\sum_{\boldsymbol{e}^{\prime} \sim \mathbf{0}} \mathrm{e}^{\lambda \boldsymbol{\ell} \cdot \boldsymbol{e}^{\prime}}}=: \tilde{p}_{\boldsymbol{e}}
$$

and $\tilde{\mathrm{P}}\left(X_{n+1}=\boldsymbol{y} \mid X_{n}=\boldsymbol{x}\right)=0$ if $\boldsymbol{y}$ is not a nearest neighbor of $\boldsymbol{x}$. The jump rate of $\boldsymbol{Y}$ at site $\boldsymbol{x}$ is equal to $\exp \left(-\beta C_{\boldsymbol{x}}\right)$ and the holding times are independent, exponentially distributed with mean $\exp \left(\beta C_{\boldsymbol{x}}\right)$. The Markov chain $\boldsymbol{X}$ is quite simple, it is the random walk on $\mathbb{Z}^{d}$ with drift

$$
d(\lambda)=\frac{1}{\sum_{k=1}^{d} \cosh \left(\lambda \ell_{k}\right)}\left(\sinh \left(\lambda \ell_{k}\right)\right)_{1 \leq k \leq d} .
$$

It is plain that, for the random walk,

$$
\frac{X_{n}}{n} \longrightarrow \boldsymbol{d}(\lambda) \quad \tilde{\mathrm{P}}-\text { a.s. }
$$

so directional transience is clear, and the law of large numbers for $\boldsymbol{Y}$ reduces to studying the clock process, which takes care of the jump times. As can be seen from (4), below, the process considered here is a generalization of the so-called random walk in a random scenery, or the random walk subordinated to a renewal process, which are used as effective models for 
anomalous diffusions. The difference is essentially that the environment (i.e. the field of jump rates) here has some space correlations, which are short range. It is also related to the trap model considered in the analysis of the ageing phenomenon introduced in [5]: the ageing of this model has been studied in detail; see [2] for a recent review.

For a fixed $\omega, \mathrm{P}_{\omega}$ is called the quenched law and we define the annealed law $\hat{\mathrm{P}}$ by

$$
\hat{\mathrm{P}}=\mathrm{P} \times \mathrm{P}_{\omega} \text {. }
$$

Of course, statements which hold $\hat{\mathrm{P}}$-a.s., equivalently hold $\mathrm{P}_{\omega}$-a.s. for $\mathrm{P}$-almost every (P-a.e.) environment.

Finally, we stress that we assume that $d \geq 1$ in this paper. The case in which $d=1$ is special since the critical threshold $p_{\mathrm{c}}(1)=1$. Moreover, specific techniques are available in one dimension (see, e.g. [20] for a survey); however, we will stay as much as possible with techniques that apply for all $d$.

Our first result is the law of large numbers.

Theorem 1. (Law of large numbers.) For any $\lambda \geq 0$ and any $\beta \geq 0$, as $t \rightarrow+\infty$,

$$
\frac{Y_{t}}{t} \rightarrow \boldsymbol{v}(\lambda, \beta) \quad \hat{\mathrm{P}} \text {-a.s. }
$$

where

$$
\boldsymbol{v}(\lambda, \beta)=\left(\mathrm{E}\left(\exp \left(\beta C_{\mathbf{0}}\right)\right)\right)^{-1} \boldsymbol{d}(\lambda) .
$$

In particular, $\boldsymbol{v}(\lambda, \beta)=0$ if $\beta>\xi$ or $\lambda=0$, though $\boldsymbol{v}(\lambda, \beta) \cdot \boldsymbol{\ell}>0$ if $\beta<\xi$ and $\lambda \neq 0$.

As considered in [14], for the case in which $\lambda=0$, slowdowns occur for a large disorder intensity $\beta$, when the walk gets trapped on large percolation clusters. This behavior is reminiscent of the biased random walk on the supercritical percolation infinite cluster [4], [19], where ballistic or subballistic regimes take place according to the parameter values. The slowdowns in our paper have a similar nature to those in some one-dimensional random walks in random environments; see [9], [17], and [18]. Moreover, as in the one-dimensional case, we obtain explicit values for the rate of escape here, a rather unusual fact in larger dimensions. More drastic (logarithmic) slowdowns were also found for an unbiased walker on a moon crater landscape in [6] and [7], or diffusions in random potentials [10], but in these models the behavior at small disorder is qualitatively different from the behavior without disorder.

The next result contains extra information on the subballistic behavior.

Theorem 2. (Subballistic regime.) Let $\beta \geq \xi$.

1. For any $d \geq 1$ and $\lambda>0$, as $t \rightarrow+\infty$,

$$
\frac{\ln \left|Y_{t}\right|}{\ln t} \rightarrow \frac{\xi}{\beta} \quad \hat{\mathrm{P}}-\text { a.s. }
$$

2. If $\lambda=0$, for any $d \geq 2$, we have

$$
\limsup _{t \rightarrow+\infty} \frac{\ln \left|Y_{t}\right|}{\ln t}=\frac{\xi}{2 \beta} \quad \hat{\mathrm{P}} \text {-a.s. }
$$

3. If $d=1$ and $\lambda=0$, we have

$$
\limsup _{t \rightarrow+\infty} \frac{\ln \left|Y_{t}\right|}{\ln t}=\frac{1}{2}\left(\frac{\beta}{2 \xi}+\frac{1}{2}\right)^{-1} \hat{\mathrm{P}} \text {-a.s. }
$$


Hence, the spread of the random walks in random environments scales algebraically with time in all cases. Note that in the isotropic cases, $\lambda=0$, the slowdown is larger for $d=1$ than for $d \geq 2$. This will appear in the proof as a consequence of the strong recurrence of the simple random walk $\boldsymbol{X}$ in the one-dimensional case. Note that our results are only on the logarithmic scale, though the scaling limit has been obtained for the isotropic trap model, in dimension $d=1$ (see, e.g. [2]) and dimension $d \geq 2$ [3] with a limit given, if the disorder is strong, by the time change of a Brownian motion by the inverse of a stable subordinator (fractional kinetics). Though we believe that the scaling limit of our model without drift $(\lambda=0)$ is the same, we could not obtain more precise results because of the presence of correlations in the medium. Moreover, the case of a drift, $\lambda \neq 0$, has not been considered in the literature, except for $d=1$ with renormalization group arguments [13].

To complete the picture, we end with the diffusive case. (Recall that $\beta<\xi$ is sufficient for $\mathrm{E}\left(\exp \left(\beta C_{\mathbf{0}}\right)\right)<\infty$.)

Theorem 3. (Diffusive case regime.) Assume that $\lambda=0$ and that $\mathrm{E}\left(\exp \left(\beta C_{\mathbf{0}}\right)\right)<\infty$. Then, we have a quenched invariance principle for the rescaled process $\boldsymbol{Z}^{\varepsilon}=\left(Z_{t}^{\varepsilon}\right)_{t \geq 0}$, $Z_{t}^{\varepsilon}=\varepsilon^{1 / 2} Y_{\varepsilon^{-1} t}$ : for almost every $\omega$, as $\varepsilon \searrow 0$, the family of processes $\boldsymbol{Z}^{\varepsilon}$ converges in law under $\mathrm{P}_{\omega}$ in the Skorokhod topology to the d-dimensional Brownian motion with diffusion matrix $\boldsymbol{\Sigma}=\left(d \times \mathrm{E}\left(\exp \left(\beta C_{\mathbf{0}}\right)\right)\right)^{-1} \quad \boldsymbol{I}_{d}$ (where $\boldsymbol{I}_{d}$ denotes the identity matrix of dimension $d$ ). Moreover,

$$
\limsup _{t \rightarrow+\infty} \frac{\ln \left|Y_{t}\right|}{\ln t}=\frac{1}{2} \quad \text { a.s. }
$$

For the proof of our results, we will take the point of view of the environment seen from the walker. It turns out that the 'static' environmental distribution is invariant for the dynamics. Hence, the environment is always at equilibrium.

The paper is organized as follows. In Section 2 we introduce the basic ingredients for our analysis and we prove the law of large numbers of Theorem 1. Section 3 is devoted to the subballistic regime and contains the proofs of Theorems 2 and 3. Finally, in Section 4 we present some concluding remarks.

\section{Preliminaries and the proof of Theorem 1}

For $\boldsymbol{x} \in \mathbb{Z}^{d}, T^{x}$ will denote the space shift with vector $\boldsymbol{x}$. We will also consider the time shift $\theta$.

\subsection{The skeleton and clock process of $Y$}

The sequence $\left(S_{n} ; n \geq 0\right)$ of jump times of the Markov process $\boldsymbol{Y}$ with right-continuous paths is defined by $S_{0}=0<S_{1}<S_{2}<\cdots, Y_{t}=Y_{S_{n}}$ for $t \in\left[S_{n}, S_{n+1}\right)$, and $Y_{S_{n+1}} \neq Y_{S_{n}}$. The skeleton of $\boldsymbol{Y}$ is the sequence $\boldsymbol{X}$ given by $X_{n}=Y_{S_{n}}, n \geq 0$. As mentioned above, the skeleton $\boldsymbol{X}$ of $\boldsymbol{Y}$ is the simple random walk with drift. For any $\boldsymbol{x}$ in $\mathbb{Z}^{d}$, the jump rate of $\left(Y_{t}\right)_{t \geq 0}$ at $\boldsymbol{x}$ is $\exp \left(-\beta C_{\boldsymbol{x}}\right)$. Hence, the time $S_{n}$ of the $n$th jump is the sum of $n$ independent random variables with exponential distribution with mean $\exp \left(\beta C_{X_{i}}\right), i=1, \ldots n$. This means that the sequence $\varepsilon=\left(\varepsilon_{i}\right)_{i \in \mathbb{N}}$, where $\varepsilon_{i}=\exp \left(-\beta C_{X_{i}}\right)\left(S_{i+1}-S_{i}\right)$, is, under the quenched law and then also under the annealed law, a sequence of i.i.d. exponential variables with mean 1 , with $\mathscr{E}$ and $\boldsymbol{X}$ independent. The law of this sequence will be denoted by $Q\left(Q=\mathscr{E x p}(1)^{\otimes \mathbb{N}}\right.$, where $\operatorname{Exp}(1)$ denotes the mean 1 exponential law). For any $n$ in $\mathbb{N}$, the time $S_{n}$ of the $n$th jump 
is given by

$$
S_{n}=\sum_{i=0}^{n-1} \varepsilon_{i} \exp \left(\beta C_{X_{i}}\right)
$$

This sequence can be viewed as a step function $S_{t}:=S_{[t]}$, where [·] is the integer part, and we also define its generalized inverse $S^{-1}$ : for any $t \geq 0$,

$$
S^{-1}(t)=n \quad \Longleftrightarrow \quad S_{n} \leq t<S_{n+1} \text {. }
$$

We observe that $S_{n} \rightarrow \infty$ as $n \rightarrow \infty \mathrm{P}_{\omega}$-a.s. for all $\omega$, making the function $S^{-1}$ defined on the whole of $\mathbb{R}_{+}$. Then, $\mathrm{P}_{\omega}$-a.s.,

$$
X_{S^{-1}(t)}=Y(t) \text { for all } t \geq 0,
$$

and, therefore, the process $S^{-1}$ is called the clock process.

Conversely, let $\mathcal{E}, \boldsymbol{X}$, and $\omega$ be independent, with distribution $\mathrm{Q}, \tilde{\mathrm{P}}$, and $\mathrm{P}$, respectively, defined on some new probability space. Then, fixing $\lambda$ and viewing $\beta$ as a parameter, by (4) and (5) we construct, on this new probability space, a coupling of the processes $\boldsymbol{Y}=\boldsymbol{Y}^{(\beta)}$ for all $\beta \in \mathbb{R}$. The coupling has the properties that the skeleton is the same for all $\beta$ and that the clock processes are such that, for $\beta \geq \beta^{\prime}$ and $t \geq 0$,

$$
S^{-1}(\beta ; t) \leq S^{-1}\left(\beta^{\prime} ; t\right) \text {. }
$$

\subsection{The environment seen from the walker}

Depending on the time being discrete or continuous, we consider the processes $\left(\tilde{\omega}_{n}\right)_{n \in \mathbb{N}}$ and $\left(\hat{\omega}_{t}\right)_{t \geq 0}$ defined by

$$
\tilde{\omega}_{n}=T^{X_{n}} \omega \quad \text { and } \quad \hat{\omega}_{t}=T^{Y_{t}} \omega=\tilde{\omega}_{S^{-1}(t)}
$$

for $n \geq 0$ and $t \geq 0$. We start with the case of discrete time.

Lemma 1. Under $\hat{\mathrm{P}},\left(\tilde{\omega}_{i}\right)_{i \in \mathbb{N}}$ is a stationary ergodic Markov chain. The same holds for $\left(\tilde{\omega}_{i}, \mathcal{E}_{i}\right)_{i \in \mathbb{N}}$.

Proof. As $\left(\varepsilon_{i}\right)_{i \in \mathbb{N}}$ is an i.i.d. sequence of variables independent of $\tilde{\omega}$, it is enough to prove Lemma 1 for the process $\left(\tilde{\omega}_{i}\right)_{i \in \mathbb{N}}$. Under $\hat{\mathrm{P}}$ or $\mathrm{P}_{\omega},\left(\tilde{\omega}_{i}\right)_{i \in \mathbb{N}}$ is Markovian with transition kernel $R$, defined, for any bounded function $f$, by

$$
R f(\omega)=\sum_{\boldsymbol{e} \sim \mathbf{0}} \tilde{p}_{\boldsymbol{e}} f\left(T^{\boldsymbol{e}} \omega\right) \quad \text { for all } \omega \in \Omega,
$$

and initial distribution $\mathrm{P}$ or, respectively, $\delta_{\omega}$. The transitions of $\left(\tilde{\omega}_{i}\right)_{i \in \mathbb{N}}$ do not depend on $\omega$, as those of $\boldsymbol{X}$, and, in this sense, the sequence is itself a random walk. Since P is invariant by translation,

$$
\hat{\mathrm{E}}\left(f\left(\tilde{\omega}_{1}\right)\right)=\int \sum_{\boldsymbol{e} \sim \mathbf{0}} \tilde{p}_{\boldsymbol{e}} f\left(T^{\boldsymbol{e}} \omega\right) \mathrm{dP}=\sum_{\boldsymbol{e} \sim \mathbf{0}} \tilde{p}_{\boldsymbol{e}} \int f\left(T^{\boldsymbol{e}} \omega\right) \mathrm{dP}=\mathrm{E}(f(\omega)),
$$

showing that $\mathrm{P}$ is an invariant measure for $\left(\tilde{\omega}_{i}\right)_{i \in \mathbb{N}}$.

We will use $\mathcal{F}$ to denote the product $\sigma$-field on $\Omega^{\mathbb{N}}$ and, for any $k \geq 0, \mathcal{F}_{k}$ will denote the $\sigma$-field generated by the first $k$ coordinates. Note that $\theta$ is measurable and preserves the law of $\tilde{\omega}$ under $\hat{\mathrm{P}}$. We have to prove that the invariant $\sigma$-field $\Sigma:=\left\{A \in \mathcal{F}, \mathbf{1}_{A}(\tilde{\omega})=\mathbf{1}_{A}(\theta \tilde{\omega}) \hat{\mathrm{P}}\right.$-a.s. $\}$ is trivial. Let $Y$ be a $\Sigma$-measurable bounded random variable on $\Omega^{\mathbb{N}}$. We have to show that $Y$ is $\hat{\mathrm{P}}$-a.s. constant. 
Define, for all $\omega$ in $\Omega, h_{Y}(\omega):=\mathrm{E}_{\omega}(Y)$. We will study this function with standard arguments; see, e.g. Chapter 17.1 .1 of [12]. Using the Markov property and the $\theta$-invariance of $Y$, we can show that

$$
h_{Y}\left(\tilde{\omega}_{k}\right)=\hat{\mathrm{E}}\left(Y \mid \mathcal{F}_{k}\right) \quad \text { for all } k \in \mathbb{N}, \hat{\mathrm{P}} \text {-a.s. }
$$

As a consequence, under $\hat{\mathrm{P}},\left(h_{Y}\left(\tilde{\omega}_{k}\right)\right)_{k \geq 0}$ is both a stationary process and an a.s. convergent martingale and, hence, it is a.s. constant. In particular,

$$
Y=h_{Y}\left(\tilde{\omega}_{0}\right) \quad \hat{\mathrm{P}} \text {-a.s., }
$$

which means that $Y$ can be considered as a function of the first coordinate alone. The next step is to show that $h_{Y}$ is $\mathrm{P}$-a.s. harmonic, that is

$$
R h_{Y}\left(\tilde{\omega}_{0}\right)=h_{Y}\left(\tilde{\omega}_{0}\right) \quad \hat{\mathrm{P}} \text {-a.s. }
$$

It is a consequence of the following computation,

$$
\begin{aligned}
R h_{Y}\left(\tilde{\omega}_{0}\right) & =\hat{\mathrm{E}}\left(h_{Y}\left(\tilde{\omega}_{1}\right) \mid \mathcal{F}_{0}\right) \quad \hat{\mathrm{P}} \text {-a.s. } \\
& =\hat{\mathrm{E}}\left(\hat{\mathrm{E}}\left(Y \mid \mathcal{F}_{1}\right) \mid \mathcal{F}_{0}\right) \quad \hat{\mathrm{P}} \text {-a.s. } \\
& =h_{Y}\left(\tilde{\omega}_{0}\right) \quad \hat{\mathrm{P}} \text {-a.s., }
\end{aligned}
$$

where the second equality is true because of (7). We will now show that $Y$ is invariant by translation in space. By the invariance of $\mathrm{P}$ and the harmonicity of $h_{Y}$, it is true that

$$
\sum_{\boldsymbol{e} \sim \mathbf{0}} \int \tilde{p}_{\boldsymbol{e}}\left(Y-Y \circ T^{\boldsymbol{e}}\right)^{2} \mathrm{dP}=0 .
$$

For every $\boldsymbol{e}$ neighbor of $\mathbf{0}, \tilde{p}_{\boldsymbol{e}}>0$, and the previous equation implies that, P-a.s., $Y=Y \circ T^{\boldsymbol{e}}$ for any $\boldsymbol{e} \sim \boldsymbol{0}$. Together with the ergodicity of $\mathrm{P}$, this shows that $Y$ is $\hat{\mathrm{P}}$-a.s. constant, and completes the proof.

As a consequence of Lemma 1 and Birkhoff's ergodic theorem, for any function $f$ in $L_{1}\left(\Omega^{\mathbb{N}}\right)$ (or $f$ nonnegative), as $n \rightarrow+\infty$,

$$
\frac{1}{n} \sum_{k=0}^{n-1} f\left(\theta^{k} \tilde{\omega}\right) \rightarrow \mathrm{E}(f) \quad \hat{\mathrm{P}} \text {-a.s. }
$$

Now, we turn to the time-continuous case, and we consider the empirical distribution $t^{-1} \int_{0}^{t} \delta_{\hat{\omega}_{s}} \mathrm{~d} s$ of the environment seen from the walker up to time $t$. Our next result is a law of large numbers for this random probability measure. For small $\beta$, the empirical distribution converges to some limit $\mathrm{P}^{0}$, which is then an invariant measure for $\left(\hat{\omega}_{t}\right)_{t \geq 0}$.

Corollary 1. If $\beta<\xi$ then, $\hat{\mathrm{P}}$-a.s., the empirical distribution of the environment seen from the walker, $t^{-1} \int_{0}^{t} \delta_{\hat{\omega}_{s}} \mathrm{~d} s$, converges weakly to $\mathrm{P}^{0}$, defined by

$$
\mathrm{dP}^{0}=\frac{\exp \left(\beta C_{\mathbf{0}}\right)}{\mathrm{E}\left(\exp \left(\beta C_{\mathbf{0}}\right)\right)} \mathrm{dP} .
$$


Proof. We need to show that $t^{-1} \int_{0}^{t} f\left(\hat{\omega}_{s}\right) \mathrm{d} s \rightarrow \int f \mathrm{dP}^{0}$ as $t \rightarrow \infty$ for all real, bounded continuous functions $f$ on $\Omega$. Since $\exp \left(\beta C_{\mathbf{0}}\right)$ is integrable when $\beta<\xi$, this follows from the convergence along the sequence $t=S_{n}, n \rightarrow \infty$. By (4), this is equivalent to

$$
\frac{n^{-1} \sum_{i=0}^{n-1} \varepsilon_{i} \exp \left(\beta C_{X_{i}}\right) f\left(\tilde{\omega}_{i}\right)}{n^{-1} \sum_{i=0}^{n-1} \varepsilon_{i} \exp \left(\beta C_{X_{i}}\right)} \longrightarrow \int_{\Omega} f \mathrm{dP}^{0}, \quad n \rightarrow \infty
$$

We first study the $\hat{\mathrm{P}}$-almost sure convergence of the denominator, i.e. of $n^{-1} S_{n}$. Define the real function $g$ on $\left(\mathbb{R}^{\mathbb{N}}, \Omega^{\mathbb{N}}\right)$ by

$$
g:\left(\left(\varepsilon_{i}\right)_{i \in \mathbb{N}},\left(\tilde{\omega}_{i}\right)_{i \in \mathbb{N}}\right) \mapsto \varepsilon_{0} \exp \left(\beta C_{\mathbf{0}}\left(\tilde{\omega}_{0}\right)\right),
$$

and note that $C_{X_{n}}=C_{\mathbf{0}}\left(\tilde{\omega}_{n}\right)$. Applying Lemma 1 and the ergodic theorem to $(\tilde{\omega}, \mathcal{E})$ and to the nonnegative function $g$, we find that $n^{-1} S_{n}$ converges $\hat{\mathrm{P}}$-a.s. to $\operatorname{E}\left(\exp \left(\beta C_{\mathbf{0}}\right)\right)$. The numerator can be studied with the same arguments, and we obtain the claim since, for $\beta<\xi$, both limits are finite.

With this in hand, we can easily complete the proof of Theorem 1.

Proof of Theorem 1. Write

$$
\frac{Y_{t}}{t}=\frac{X_{S^{-1}(t)}}{S^{-1}(t)} \frac{S^{-1}(t)}{S\left(S^{-1}(t)\right)} \frac{S\left(S^{-1}(t)\right)}{t} .
$$

Recall from (1) that the first factor on the right-hand side converges a.s. to $d(\lambda)$ as $t \rightarrow \infty$. In the proof of Corollary 1 we showed that $S\left(S^{-1}(t)\right) / S^{-1}(t) \rightarrow \mathrm{E}\left(\exp \left(\beta C_{0}\right)\right)$ a.s. for $\beta<\xi$, but clearly the result remains true for all $\beta$ (the limit is infinite for $\beta>\xi$ ). For the last factor on the right-hand side, we simply observe that

$$
\frac{S\left(S^{-1}(t)\right)}{S\left(S^{-1}(t)+1\right)} \leq \frac{S\left(S^{-1}(t)\right)}{t} \leq 1
$$

concluding that $S\left(S^{-1}(t)\right) / t$ converges $\hat{\mathrm{P}}$-a.s. to 1 if $\mathrm{E}\left(\exp \left(\beta C_{\mathbf{0}}\right)\right)<\infty$ : in this case we then conclude that $Y_{t} / t$ converges $\hat{\mathrm{P}}$-a.s. to $\boldsymbol{v}(\lambda, \beta)$ given in (2).

For the case in which $\operatorname{E}\left(\exp \left(\beta C_{0}\right)\right)=\infty$, we just use the right inequality in (8) to obtain the $\hat{\mathrm{P}}$-almost sure convergence of $Y_{t} / t$ to $v(\lambda, \beta)=0$.

\section{Subballistic regime, and the proofs of Theorems 2 and 3}

We start with a few auxiliary results.

Lemma 2. Assume that $d \geq 2$ or $\lambda>0$. Then, for any $\varepsilon>0$, there exists $\alpha>0$ such that, $\hat{\mathrm{P}}$-a.s., we eventually have

$$
\#\left\{i \leq n, C_{X_{i}}>\left(\frac{1}{\xi}-\varepsilon\right) \ln n\right\} \geq n^{\alpha},
$$

where the notation \#A denotes for the cardinality of the set A.

Proof. Define the range $R_{n}$ to be the number of points visited by $\left(X_{i}\right)_{i \in \mathbb{N}}$ during the first $n$ steps. For $\lambda>0$, there exists a constant $c_{1}>0$ such that, $\tilde{\mathrm{P}}$-a.s., $R_{n}>c_{1} n$ eventually. For 
$\lambda=0$ and $d \geq 2$, it is well known (see Chapter 21 of [15]) that there exists a constant $c_{2}$ such that, $\tilde{\mathrm{P}}$-a.s., $R_{n}>c_{2} n / \ln n$ eventually (when $d \geq 3$, the walk is transient and the correct order of $R_{n}$ is $n$ ). In all cases there exists a constant $c_{3}>0$ such that, under the assumptions of Lemma 2 , we have, $\tilde{\mathrm{P}}$-a.s., $R_{n}>c_{3} n / \ln n$ eventually. For a fixed $n$ in $\mathbb{N}$, we recursively define the time $T_{i}^{n}$ by

$$
\begin{gathered}
T_{0}^{n}=0 \\
T_{i}^{n}=\inf \left\{T_{i-1}^{n}<k \leq n,\left|X_{k}-X_{T_{j}^{n}}\right|>2\left(\frac{1}{\xi}-\varepsilon\right) \ln n \text { for all } j<i\right\} \quad \text { for all } i \geq 1, \\
\inf \varnothing=+\infty
\end{gathered}
$$

Note that the balls with center $X_{T_{j}^{n}}$ and radius $\left(\xi^{-1}-\varepsilon\right) \ln n$ are pairwise disjoint, and define $K_{n}$ to be the number of such balls, i.e.

$$
K_{n}=\max \left\{i \geq 0: T_{i}^{n}<+\infty\right\}
$$

As the cardinality of these balls is $c_{4} \ln ^{d} n$ (for some $c_{4}>0$ ), it follows from the previous discussion on the range that, $\tilde{\mathrm{P}}$-a.s., $K_{n}>c n / \ln ^{d+1} n$ eventually, where $c$ denotes a positive constant. From now on we fix a path $\left(X_{i}\right)_{i \geq 0}$ such that $K_{n}>c n / \ln ^{d+1} n$ eventually. In the rest of the proof, we take $n$ large enough so that the inequality holds. Then,

$$
\begin{aligned}
& \mathrm{P}\left(\#\left\{i \leq K_{n}, C_{X_{T_{i}^{n}}} \leq\left(\frac{1}{\xi}-\varepsilon\right) \ln n\right\} \geq K_{n}-n^{\alpha}\right) \\
& \quad=\mathrm{P}\left(\text { there exists } I \subset\left\{1, \ldots K_{n}\right\}, \# I=K_{n}-\left[n^{\alpha}\right] \text { : for all } i \in I, C_{X_{T_{i}^{n}}} \leq\left(\frac{1}{\xi}-\varepsilon\right) \ln n\right) \\
& \quad \leq \sum_{I \subset\left\{1, \ldots K_{n}\right\}, \# I=K_{n}-\left[n^{\alpha}\right]} \mathrm{P}\left(\text { for all } i \in I, C_{X_{T_{i}^{n}}} \leq\left(\frac{1}{\xi}-\varepsilon\right) \ln n\right) .
\end{aligned}
$$

For all $j$ such that $0 \leq j \leq K_{n}-n^{\alpha}, B_{i}^{n}$ denotes the ball with center $X_{T_{i}^{n}}$ and radius $\left(\xi^{-1}-\varepsilon\right) \ln n$. The event $\left.\left\{C_{X_{T_{i}^{n}}} \leq\left(\xi^{-1}-\varepsilon\right) \ln n\right)\right\}$ is $\sigma\left\{\omega_{x}, \boldsymbol{x} \in B_{i}^{n}\right\}$-measurable. As the balls $B_{i}^{n}$ are disjoint and the environment is i.i.d.,

$$
\begin{aligned}
\mathrm{P}(\# & \left.\left\{i \leq K_{n}, C_{X_{T_{i}^{n}}} \leq\left(\frac{1}{\xi}-\varepsilon\right) \ln n\right\} \geq K_{n}-n^{\alpha}\right) \\
& \leq\left(\begin{array}{c}
K_{n} \\
{\left[n^{\alpha}\right]}
\end{array}\right)\left(1-\mathrm{P}\left(C_{\mathbf{0}}>\left(\frac{1}{\xi}-\varepsilon\right) \ln n\right)\right)^{K_{n}-\left[n^{\alpha}\right]} \\
& \leq c_{5} n^{n^{\alpha}}\left(1-n^{-(1-\varepsilon \xi)+o(1)}\right)^{c n / \ln ^{d+1} n-n^{\alpha}}
\end{aligned}
$$

for some suitable constant $c_{5}>0$. We now choose $\alpha<\min (1, \varepsilon \xi)$, so that

$$
\sum_{n} \mathrm{P}\left(\#\left\{i \leq K_{n}, C_{X_{T_{i}}} \leq\left(\frac{1}{\xi}-\varepsilon\right) \ln n\right\} \geq K_{n}-n^{\alpha}\right)<\infty .
$$

We conclude using Borel-Cantelli's lemma.

Lemma 3. Assume that $\beta>\xi$. For $d \geq 2$ or $\lambda>0$, we have $\liminf _{n} \ln S_{n} / \ln n \geq \beta / \xi \hat{\mathrm{P}}$-a.s. 
Proof. Let $\eta$ be a positive real number. With $\varepsilon:=\eta / \beta$, from Lemma 2, there exists $\alpha>0$ such that, $\tilde{\mathrm{P}} \otimes \mathrm{P}$-a.s., there exists a natural number $N=N(X, \omega)$ such that, for $n>N$, the set $I=\left\{i \leq n, C_{X_{i}}>\left(\xi^{-1}-\varepsilon\right) \ln n\right\}$ has cardinality $\# I \geq n^{\alpha}$. For $n>N$,

$$
\begin{aligned}
Q\left(S_{n}<n^{\beta / \xi-\eta}\right) & \leq Q\left(\mathcal{E}_{i} \exp \left(\beta C_{X_{i}}\right)<n^{\beta / \xi-\eta}, i \in I\right) \\
& \leq Q\left(\mathcal{E}_{1} \exp \left(\beta C_{X_{1}}\right)<n^{\beta / \xi-\eta}\right)^{n^{\alpha}} \\
& \leq Q\left(\mathcal{E}_{1}<n^{\beta \varepsilon-\eta}\right)^{n^{\alpha}} \\
& =\left(1-\mathrm{e}^{-1}\right)^{n^{\alpha}} .
\end{aligned}
$$

From the previous inequality, we find that $Q\left(S_{n}<n^{\beta / \xi-\eta}\right)$ is the general term of a convergent series, and we can use Borel-Cantelli's Lemma to conclude.

Lemma 4. Assume that $\beta>\xi$. For $d \geq 1$ and $\lambda \geq 0$, we have, $\hat{\mathrm{P}}-$ a.s., $\lim \sup _{n} \ln S_{n} / \ln n \leq$ $\beta / \xi$.

Proof. For any $\alpha$ in $(0,1)$, by subadditivity we have $(u+v)^{\alpha} \leq u^{\alpha}+v^{\alpha}$ for all positive $u$ and $v$, and then

$$
S_{n}^{\alpha} \leq \sum_{i=1}^{n} \varepsilon_{i}^{\alpha} \exp \left(\alpha \beta C_{X_{i}}\right)
$$

Now, define the function $f_{\alpha}$ as follows:

$$
\begin{aligned}
f_{\alpha}: & \left(\mathbb{R}^{\mathbb{N}}, \Omega^{\mathbb{N}}\right) \rightarrow \mathbb{R}, \\
& \left(\left(\mathcal{E}_{i}\right)_{i \in \mathbb{N}},\left(\tilde{\omega}_{i}\right)_{i \in \mathbb{N}}\right) \rightarrow \varepsilon_{0}^{\alpha} \exp \left(\alpha \beta C_{\mathbf{0}}\left(\tilde{\omega}_{0}\right)\right) .
\end{aligned}
$$

Applying Lemma 1 and the ergodic theorem to $(\tilde{\omega}, \mathcal{E})$ with the nonnegative function $f_{\alpha}$, we obtain, for any $\alpha$ such that $\alpha \beta<\xi$,

$$
\limsup _{n \rightarrow+\infty} \frac{S_{n}^{\alpha}}{n} \leq \lim _{n \rightarrow+\infty} \frac{\sum_{i=1}^{n} \mathcal{E}_{i}^{\alpha} \exp \left(\alpha \beta C_{X_{i}}\right)}{n}=\mathrm{E}_{\mathrm{Q}}\left(\mathcal{E}_{1}^{\alpha}\right) \times \mathrm{E}\left(\exp \left(\alpha \beta C_{\mathbf{0}}\right)\right)<\infty \quad \text { a.s. }
$$

Therefore,

$$
\limsup _{n \rightarrow+\infty} \frac{\ln S_{n}}{\ln n}<\frac{1}{\alpha} .
$$

Since $\alpha$ is arbitrary in $(0, \xi / \beta)$, the proof is complete.

The following two lemmas deal with the one-dimensional case. Note that when $d=1$, for all $n>0$,

$$
\mathrm{P}(C \geq n)=p \sum_{k=0}^{n-1} p^{k} p^{n-1-k}=n p^{n}
$$

and, as a consequence, $\xi=-\ln p$.

Lemma 5. Assume that $\beta>\xi$. For $d=1$ and $\lambda=0$, we have, $\hat{\mathrm{P}}-$ a.s., $\lim \sup _{n} \ln S_{n} / \ln n \leq$ $\beta / 2 \xi+\frac{1}{2}$.

Proof. Here we need to relabel our sequence of exponential variables $\left(\varepsilon_{i} ; i \geq 0\right)$. For $y \in \mathbb{Z}$ and $k \in \mathbb{N}$, define $\varepsilon_{y, k}$ by

$$
\mathcal{E}_{y, k}=\mathcal{E}_{i} \quad \text { with } i \text { such that } \quad X_{i}=y, \#\left\{j: 0 \leq j \leq i, X_{j}=y\right\}=k,
$$


i.e. the exponential corresponding to the $k$ th passage at $y$. These new variables are a.s. well defined when $d=1$ and $\lambda=0$, and it is not difficult to see that the sequence $\left(\varepsilon_{y, k}\right)_{y \in \mathbb{Z}, k \in \mathbb{N}}$ is i.i.d. with mean 1 exponential distribution, and independent of $\boldsymbol{X}$ and $\omega$. The number of visits of the walk to a site $y$ at time $n$ will be denoted by $\theta(n, y)$. We can rewrite $S_{n}$ in the following way:

$$
S_{n}=\sum_{i=0}^{n-1} \exp \left(\beta C_{X_{i}}\right) \mathcal{E}_{i}=\sum_{y \in \mathbb{Z}} \exp \left(\beta C_{y}\right)\left(\sum_{k=0}^{\theta(n, y)-1} \mathcal{E}_{y, k}\right)
$$

Note that, for any $\eta>0, \tilde{\mathrm{P}}$-a.s. for large enough $n$,

$$
\theta(n, y)=0 \quad \text { for all } y>n^{1 / 2+\eta}
$$

(see, e.g. Theorem 5.7 of [15]). As a consequence, we obtain, $\tilde{\mathrm{P}}$-a.s., for any positive $\alpha<1$ and for large enough $n$,

$$
S_{n}^{\alpha} \leq \sum_{y=-n^{-1 / 2+\eta}}^{n^{1 / 2+\eta}} \exp \left(\alpha \beta C_{y}\right)\left(\sum_{k=0}^{\theta(n, y)-1} \mathcal{E}_{y, k}\right)^{\alpha} .
$$

Here and below, the sum $\sum_{y=a}^{b}$ with real numbers $a<b$ ranges over all $y \in \mathbb{Z}$ with $a \leq y \leq b$. Now note that, $\tilde{\mathrm{P}}$-a.s., for any $v>0$ and large enough $n$,

$$
\sup \{\theta(n, y), y \in \mathbb{Z}\}<n^{1 / 2+v}
$$

(see, e.g. Theorem 11.3 of [15]), and we obtain for such $n$,

$$
\frac{1}{2 n^{1 / 2+\eta} n^{(1 / 2+\nu) \alpha}} S_{n}^{\alpha} \leq \frac{1}{2 n^{1 / 2+\eta}} \sum_{y=-n^{-1 / 2+\eta}}^{n^{1 / 2+\eta}} \exp \left(\alpha \beta C_{y}\right)\left(\frac{1}{n^{1 / 2+v}} \sum_{k=0}^{n^{1 / 2+v}} \mathcal{E}_{y, k}\right)^{\alpha} .
$$

For any $y$ in $\mathbb{Z}$ and $n$ in $\mathbb{N}$, we define

$$
u_{y, n}=\frac{1}{n^{1 / 2+v}} \sum_{k=0}^{n^{1 / 2+v}} \varepsilon_{y, k} .
$$

Fix $\mu>0$. According to the large deviation principle for i.i.d. sequences, there exists $I_{\mu}>0$ such that, for any $y$ in $\mathbb{Z}$ and any $n$ in $\mathbb{N}$,

$$
\mathrm{Q}\left(\left|u_{y, n}-1\right|>\mu\right) \leq \exp \left(-I_{\mu} n^{1 / 2+v}\right) .
$$

Using the independence of the $\left(\mathcal{E}_{y, k}\right)_{y \in \mathbb{Z}, k \in \mathbb{N}}$, it is easy to check that Q (there exists $y \in$ $\left[-n^{1 / 2+\eta}, n^{1 / 2+\eta}\right]$ and $\left.\left|u_{y, n}-1\right|>\mu\right)$ is the general term of a convergent series, and using Borel-Cantelli's lemma, we obtain, Q-a.s., for large enough $n$ and for any $-n^{1 / 2+\eta}<y<$ $n^{1 / 2+\eta}$,

$$
\left|u_{y, n}-1\right|<\mu \text {. }
$$

From the ergodicity of the environment, it is true that P-a.s., as $n \rightarrow+\infty$,

$$
\frac{1}{2 n^{1 / 2+\eta}} \sum_{y=-n^{-1 / 2+\eta}}^{n^{1 / 2+\eta}} \exp \left(\alpha \beta C_{y}\right) \rightarrow \mathrm{E}\left(\exp \left(\alpha \beta C_{\mathbf{0}}\right)\right)
$$


Now using (11), (12), and (13), we find that, for any $\alpha<\xi / \beta$, there exists $M<+\infty$ such that, P-a.s., for large enough $n$,

$$
S_{n}<M n^{1 / 2 \alpha+\eta / \alpha+1 / 2+v}
$$

Since the last inequality is true for $\eta$ and $\mu$ arbitrary small and $\alpha$ arbitrary close to $\xi / \beta$, the proof is complete.

Lemma 6. Assume that $\beta>\xi$. For $d=1$ and $\lambda=0$, we have, $\hat{\mathrm{P}}-a . s$,

$$
\liminf _{n \rightarrow \infty} \frac{\ln S_{n}}{\ln n} \geq \frac{\beta}{2 \xi}+\frac{1}{2} .
$$

Proof. Let $\eta$ and $v$ be two positive real numbers. As a consequence of (9) and (10), $\tilde{\mathrm{P}}$-a.s., for large enough $n$, at least $n^{1 / 2-\nu \xi / 4}$ sites are visited more than $n^{1 / 2-\eta}$ times; we will denote the set of those sites by $O_{n}$. Now fix a path $\left(X_{i}\right)_{i \geq 0}$ such that, for all $n \geq 0$, \# $O_{n} \geq n^{1 / 2-v \xi / 4}$. As in the proof of Lemma 3, we can choose a family of

$$
\alpha_{n}:=\frac{n^{1 / 2-\nu \xi / 4}}{(1 / 2)(1 / \xi-v) \ln n}
$$

points $\left(y_{i}\right)_{i \leq \alpha_{n}}$ in $O_{n}$ such that the intervals $\left(I_{i}\right)_{i \leq \alpha_{n}}$ centered in $\left(y_{i}\right)_{i \leq \alpha_{n}}$ and of length $\frac{1}{2}\left(\xi^{-1}-\right.$ $\nu$ ) are disjoint. We say that such an interval is open if all its sites are open, and closed otherwise. Using the fact that the $\left(I_{i}\right)_{i \leq \alpha_{n}}$ are disjoint, we obtain,

$$
\begin{aligned}
\mathrm{P}\left(I_{i} \text { is closed for all } i \leq \alpha_{n}\right) & \leq\left(1-n^{-1 / 2(1-\nu \xi)+o(1)}\right)^{\alpha_{n}} \\
& \leq \exp \left(-n^{\nu \xi / 4+o(1)}\right) .
\end{aligned}
$$

Borel-Cantelli's lemma implies that, P-a.s., for large enough $n$, the following property holds: there exists at least one site visited more than $n^{1 / 2-\eta}$ times, and this site belongs to a cluster of size greater than $\frac{1}{2}\left(\xi^{-1}-v\right) \ln n$; we will denote this site by $\tilde{y}_{n}$. Therefore,

$$
S_{n} \geq \sum_{i=0}^{n^{1 / 2-\eta}} n^{\beta / 2 \xi-\nu \beta} \mathcal{E}_{\tilde{y}_{n}, i}
$$

Using the large deviation upper bound similarly to the lines below (11), we obtain from the last inequality, $\hat{\mathrm{P}}$-a.s., for large enough $n$,

$$
S_{n} \geq \frac{1}{2} n^{1 / 2+\beta / 2 \xi-v \beta-\eta} .
$$

Since $v$ and $\eta$ can be chosen arbitrary small, this last inequality completes the proof.

Proof of Theorem 2. We first assume that $\beta>\xi$. From Lemma 3 and Lemma 4, we know that, under the assumptions of parts 1 or 2 ,

$$
\lim _{n \rightarrow+\infty} \frac{\ln S_{n}}{\ln n}=\frac{\beta}{\xi} \quad \hat{\mathrm{P}} \text {-a.s. }
$$

From the inequalities

$$
\frac{\ln S\left(S^{-1}(t)\right)}{\ln S^{-1}(t)} \leq \frac{\ln t}{\ln S^{-1}(t)}<\frac{\ln S\left(S^{-1}(t)+1\right)}{\ln S^{-1}(t)},
$$


we deduce that, $\hat{\mathrm{P}}$-a.s.,

$$
\lim _{t \rightarrow+\infty} \frac{\ln t}{\ln S^{-1}(t)}=\frac{\beta}{\xi} .
$$

Applying the same arguments as above, we deduce from Lemma 5 and Lemma 6 that, under the assumptions of part 3 ,

$$
\lim _{t \rightarrow+\infty} \frac{\ln t}{\ln S^{-1}(t)}=\frac{\beta}{2 \xi}+\frac{1}{2} \quad \hat{\text { P-a.s. }}
$$

Now write

$$
\frac{\ln \left|Y_{t}\right|}{\ln t}=\frac{\ln \left|X_{S^{-1}(t)}\right|}{\ln S^{-1}(t)} \frac{\ln S^{-1}(t)}{\ln t} .
$$

To conclude the $\beta>\xi$ case, note that, under the assumptions of part $1, \ln \left|X_{n}\right| / \ln n$ converges $\tilde{\mathrm{P}}$-a.s. to 1 and, under the assumptions of parts 2 and 3, $\tilde{\mathrm{P}}$-a.s., $\lim \sup _{n \rightarrow+\infty} \ln \left|X_{n}\right| / \ln n=\frac{1}{2}$, by the law of iterated logarithms.

To extend the results to the border case, $\beta=\xi$, we use property (6) of the coupling, which implies that the long-time limit of $\ln \left|Y_{t}\right| / \ln t$ is nonincreasing in $\beta$. This completes the proof of part 1 with $\beta=\xi$. For the other parts, we use property (3), which we will prove independently below. Again, the results claimed for $\beta=\xi$ in parts 2 and 3 follow from the monotonicity of the coupling.

Proof of Theorem 3. First observe that, when $\lambda=0$,

$$
f\left(Y_{t}\right)-\int_{0}^{t}(2 d)^{-1} \exp \left(-\beta C_{Y_{s}}\right) \sum_{\boldsymbol{e} \sim \mathbf{0}}\left(f\left(Y_{s}+\boldsymbol{e}\right)-f\left(Y_{s}\right)\right) \mathrm{d} s
$$

is a $\mathrm{P}_{\omega}$-martingale for continuous and bounded $f$. Then, for all $\omega$, the process $\boldsymbol{Y}$ is a square integrable martingale under the quenched law $\mathrm{P}_{\omega}$. Its bracket is the unique process $\langle\boldsymbol{Y}\rangle$, taking its values in the space of nonnegative symmetric $d \times d$ matrices such that $\boldsymbol{Y}_{t} \boldsymbol{Y}_{t}^{\top}-\langle\boldsymbol{Y}\rangle_{t}$ is a martingale and $\langle\boldsymbol{Y}\rangle_{0}=0$. (Here ${ }^{T}$, denotes the transposition.) We easily compute

$$
\langle\boldsymbol{Y}\rangle_{t}=\left(\int_{0}^{t} \exp \left(-\beta C_{Y_{s}}\right) \mathrm{d} s\right) d^{-1} \boldsymbol{I}_{d} .
$$

By Corollary 1, we see that the bracket $\boldsymbol{Z}^{\varepsilon}$ is such that, for all $t \geq 0$,

$$
\begin{aligned}
\left\langle\boldsymbol{Z}^{\varepsilon}\right\rangle_{t} & =\varepsilon\langle\boldsymbol{Y}\rangle_{\varepsilon^{-1} t} \\
& =\varepsilon\left(\int_{0}^{\varepsilon^{-1} t} \exp \left(-\beta C_{Y_{s}}\right) \mathrm{d} s\right) d^{-1} \boldsymbol{I}_{d} \\
& \longrightarrow t \boldsymbol{\Sigma} \quad \hat{\mathrm{P}} \text {-a.s. as } \varepsilon \searrow 0,
\end{aligned}
$$

and then in $\mathrm{P}_{\omega}$-probability for a.e. $\omega$. Let us fix such an $\omega$, and use the law $\mathrm{P}_{\omega}$. Since the martingale $\boldsymbol{Z}^{\varepsilon}$ has jumps of size $\varepsilon^{-1 / 2}$ tending to 0 and since its bracket converges to a deterministic limit, it is well known (e.g. Theorem VIII-3.11 of [8]) that the sequence $\left(\boldsymbol{Z}^{\varepsilon}, \varepsilon>0\right)$ converges to the centered Gaussian process with variance $t \boldsymbol{\Sigma}$, yielding the desired invariance principle under $\mathrm{P}_{\omega}$.

We now prove (3). Since $\lambda=0$, we have $\lim \sup _{n} \ln \left|X_{n}\right| / \ln n=\frac{1}{2} \tilde{\mathrm{P}}$-a.s., and since $\mathrm{E}\left(\exp \left(\beta C_{\mathbf{0}}\right)\right)<\infty$, it holds that $\lim _{t} \ln S^{-1}(t) / \ln t=1$ a.s. This implies the claim. 


\section{Concluding remarks}

(i) Part 2 of Theorem 2 deals with the upper limit in the subdiffusive case, $\lambda=0$ and $\beta>\xi$. We comment here on the lower limit. In dimension $d \geq 3, n^{-1 / 2}\left|X_{[n s]}\right|$ converges to a transient Bessel process, and it is not difficult to see that

$$
\limsup _{t \rightarrow \infty} \frac{\ln \left|Y_{t}\right|}{\ln t}=\lim _{t \rightarrow \infty} \frac{\ln \left|Y_{t}\right|}{\ln t}=\frac{\xi}{2 \beta}
$$

In dimension $d \leq 2, \boldsymbol{X}$ is recurrent. Then $\liminf _{t}\left|Y_{t}\right|=0$ and

$$
\liminf _{t \rightarrow \infty} \frac{\ln \left|Y_{t}\right|}{\ln t}=-\infty
$$

(ii) A natural question is: What does the environment seen from the walker look like in the subballistic case? In fact, the prominent feature is that the size of the surrounding cluster is essentially the largest one which was visited so far. Consider, for instance, the case of positive $\lambda$. We can prove that, for $\beta>\xi$ and $\varepsilon>0$,

$$
\frac{1}{t}\left|\left\{s \in[0, t]:(\ln t)^{-1} C_{Y_{s}} \in\left[\beta^{-1}-\varepsilon, \beta^{-1}+\varepsilon\right]\right\}\right| \longrightarrow 1 \quad \hat{\mathrm{P}} \text {-a.s. as } t \nearrow \infty .
$$

(iii) We end the paper with a short comment on the case when the environment is a general random field, not necessarily coming from site percolation. It is easy to check that Theorems 1 and 3 , together with their proofs, remain valid for a stationary, ergodic random field $\left(C_{\boldsymbol{x}}, \boldsymbol{x} \in\right.$ $\mathbb{Z}^{d}$ ). On the contrary, our proof of Theorem 2 uses some independence property specific to the percolation model.

\section{Acknowledgements}

We thank Marina Vachkovskaia for stimulating discussions on the model. This research was partially supported by CNRS (UMR 7599 'Probabilités et Modéles Akéatoires').

\section{References}

[1] Aizenman, M. and Barsky, D. (1987). Sharpness of the phase transition in percolation models. Commun. Math. Phys. 108, 489-526.

[2] Ben Arous, G. and C̆erný, J. (2006). Dynamics of trap models. In Mathematical Statistical Physics (Les Houches Summer School LXXXIII, 2005), Elsevier, Amsterdam.

[3] Ben Arous, G. and Černý, J. (2007). Scaling limit for trap models on $\mathbb{Z}^{d}$. Ann. Prob. 35, 2356-2384.

[4] Berger, N., Gantert, N. And Peres, Y. (2003). The speed of biased random walk on percolation clusters. Prob. Theory Relat. Fields 126, 221-242.

[5] Bouchaud, J.-P. (1992). Weak ergodicity breaking and aging in disordered systems. J. Phys. I France 2, 17051713.

[6] Bramson, M. and Durrett, R. (1988). Random walk in random environment: a counterexample? Commun. Math. Phys. 119, 199-211.

[7] DurRetT, R. (1986). Multidimensional random walks in random environments with subclassical limiting behavior. Commun. Math. Phys. 104, 87-102.

[8] JACOD, J. And ShiRyaev, A. (1987). Limit Theorems for Stochastic Processes. Springer, Berlin.

[9] Kesten, H., Kozlov, M. and Spitzer, F. (1975). A limit law for random walk in a random environment. Compositio Math. 30, 145-168.

[10] Mathieu, P. (1994). Zero white noise limit through Dirichlet forms, with application to diffusions in a random medium. Prob. Theory Relat. Fields 99, 549-580.

[11] Menshikov, M. (1986). Coincidence of critical points in percolation problems. Dokl. Akad. Nauk SSSR 288, 1308-1311 (in Russian). 
[12] Meyn, S. P. And Tweedie, R. L. (1993). Markov Chains and Stochastic Stability. Springer, London.

[13] Monthus, C. (2004). Nonlinear response of the trap model in the aging regime: exact results in the strongdisorder limit Phys. Rev. E 69, 026103.

[14] Popov, S. ANd Vachkovskaia, M. (2005). Random walk attracted by percolation clusters. Electron. Commun. Prob. 10, 263-272.

[15] RevÉsz, P. (2005). Random Walk in Random and Non-Random Environments, 2nd edn. World Scientific, Hackensack, NJ.

[16] Shen, L. (2002). Asymptotic properties of certain anisotropic walks in random media. Ann. Appl. Prob. 12, 477-510.

[17] SinaI, Y. (1982). The limit behavior of a one-dimensional random walk in a random environment. Teor. Veroyat. Primen. 27, 247-258 (in Russian).

[18] Solomon, F. (1975). Random walks in a random environment. Ann. Prob. 3, 1-31.

[19] Sznitman, A.-S. (2003). On the anisotropic walk on the supercritical percolation cluster. Commun. Math. Phys. 240, 123-148.

[20] Zeitouni, O. (2004). Random walks in random environment. In Lectures on Probability Theory and Statistics (Lecture Notes Math. 1837), Springer, Berlin, pp. 189-312. 